\title{
Le tremblement de la figure analogique chez Rabelais. Entre la bête et l'homme
}

Louise Million

\section{(2) OpenEdition}

1 Journals

Édition électronique

URL : http://journals.openedition.org/imagesrevues/385

DOI : 10.4000/imagesrevues.385

ISSN : 1778-3801

Éditeur:

Centre d'Histoire et Théorie des Arts, Groupe d'Anthropologie Historique de l'Occident Médiéval, Laboratoire d'Anthropologie Sociale, UMR 8210 Anthropologie et Histoire des Mondes Antiques

\section{Référence électronique}

Louise Million, « Le tremblement de la figure analogique chez Rabelais. Entre la bête et l'homme », Images Re-vues [En ligne], 6 | 2009, mis en ligne le 01 juin 2009, consulté le 31 janvier 2021. URL : http://journals.openedition.org/imagesrevues/385; DOI : https://doi.org/10.4000/imagesrevues.385

Ce document a été généré automatiquement le 31 janvier 2021.

Images Re-vues est mise à disposition selon les termes de la Licence Creative Commons Attribution Pas d'Utilisation Commerciale 4.0 International. 


\title{
Le tremblement de la figure analogique chez Rabelais. Entre la bête et l'homme
}

\author{
Louise Million
}

Dans son cycle romanesque, Rabelais puise généreusement dans le lexique animal. On compte deux cent soixantedix-sept occurrences de noms d'animaux dans le Pantagruel et sept cent quarantehuit dans le Quart livre. L'animal tient donc une place de choix dans l'imaginaire rabelaisien. Le plus souvent, il est pris dans un système figuratif ; il est rarement

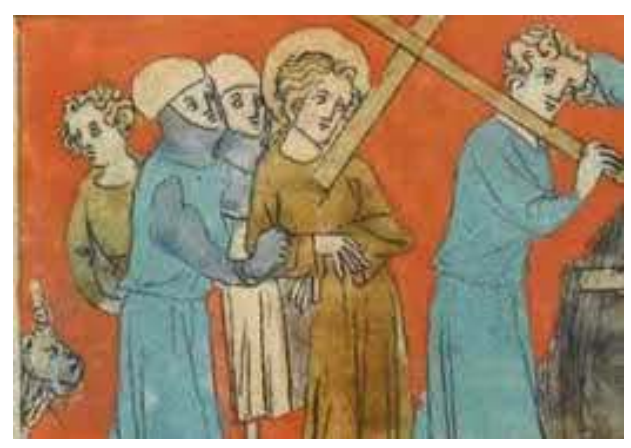
référentiel. Lazare Sainéan ${ }^{1}$ relevait déjà cette préséance de l'animal dans l'univers imaginaire de Rabelais, notamment dans l'usage des proverbes animaliers. L'emploi du proverbe marque un asservissement symbolique de l'animal. Pris dans une structure de figuration, il perd, pour ainsi dire, sa nature propre, il n'est jamais qu'au service du sens. Pourtant, Rabelais renverse cet usage lexicalisé de la parémiologie en réveillant systématiquement les images endormies. Chez lui, on n'est pas « fort comme un bœuf ", mais « comme quatre bœufs ». Ces petites distorsions redonnent continuellement vie aux images animales, leur confèrent une épaisseur certaine. Jacques Rancière parle de la «chair des mots $~^{2}$, et c'est bien cela qui semble naître sous la plume de Rabelais. Si l'on s'intéresse particulièrement à l'image animale en tant que pièce d'un système analogique, force est de s'étonner de sa capacité à résister au rôle traditionnel de l'image, à savoir l'illustration ou l'éclairage d'un signifié. Elle demeure indépendante, douée d'une animation propre. Ce caractère irréductible de l'image rabelaisienne crée des analogies singulières où les deux plans référentiels luttent sans s'abîmer dans un sens global, ce qui est le propre de l'image poétique, telle que la conçoivent Pierre Reverdy et André Breton. Il s'agit de comprendre le fonctionnement de ce système 
analogique paradoxal qui, loin de réunir les entités, nourrit les différences, tout en les confrontant, sous la contrainte de procédés littéraires forçant le rapprochement. Mue par les forces contradictoires de l'identité et de la différence, l'analogie rabelaisienne livre une image délibérément floutée de l'homme - qui demeure l'endroit de la similitude. Aussi toute image animale n'est-elle jamais qu'en devenir, puisque aucun être ne s'inscrit jamais dans une essence simple, mais toujours dans un système complexe de rapport, entre proximité et affrontement. L'homme tel que le conçoit Rabelais n'a pas de propre, sinon le rire qui appelle le mélange, le chahut, le renversement, l'éternel devenir. Il convient à présent de développer les modalités de ces devenirs de l'image animale.

\section{Le mot animal : un matériau de l'imaginaire rabelaisien}

2 Le statut de l'image animale se fait volontiers difficile à cerner, Rabelais situant celle-ci à divers degrés d'appréhension. L'animal référentiel est de fait assez rare, sinon les montures de la guerre picrocholine ou bien les fameux repas pantagruéliques du Gargantua ou du Quart livre. Servant peu la mimesis romanesque, l'animal s'inscrit plutôt dans des figures d'accumulation, de concrétion, et plus souvent encore dans des figures analogiques. Il fait rarement l'objet d'une description ${ }^{3}$; au contraire, il apparaît comme un mot suffisant, a priori évocateur, tel une touche de couleur, qui d'emblée remplirait son office dans la trame poétique (il s'agit bien alors de poéticité, pour reprendre la notion de Roman Jakobson, plutôt que de narration). L'usage des termes relevant de l'animalité dans le texte de Rabelais correspond exactement à la vision du langage chez les hommes de la Renaissance, telle que la présente Michel Foucault :

$\mathrm{Au} \mathrm{XVI}{ }^{\mathrm{e}}$ siècle, le langage réel n'est pas un ensemble de signes indépendants, uniforme et lisse où les choses viendraient se refléter comme dans un miroir pour $\mathrm{y}$ énoncer une à une leur vérité singulière. Il est plutôt chose opaque, mystérieuse, refermée sur elle-même, masse fragmentée et de point en point énigmatique, qui se mêle ici ou là aux figures du monde et s'enchevêtre à elles : tant et si bien que, toutes ensemble, elles forment un réseau de marques où chacune peut jouer, et joue en effet, par rapport à toutes les autres, le rôle de contenu ou de signe, de secret ou d'indication. Dans son être brut et historique du XVI siècle, le langage n'est pas un système arbitraire ; il est déposé dans le monde et il en fait partie à la fois parce que les choses elles-mêmes cachent et manifestent leur énigme comme un langage, et parce que les mots se proposent aux hommes comme des choses à déchiffrer. La grande métaphore du livre qu'on ouvre, qu'on épelle et qu'on lit pour connaître la nature, n'est que l'envers visible d'un autre transfert, beaucoup plus profond, qui contraint le langage à résider du côté du monde, parmi les plantes, les herbes, les pierres, et les animaux. ${ }^{4}$ 


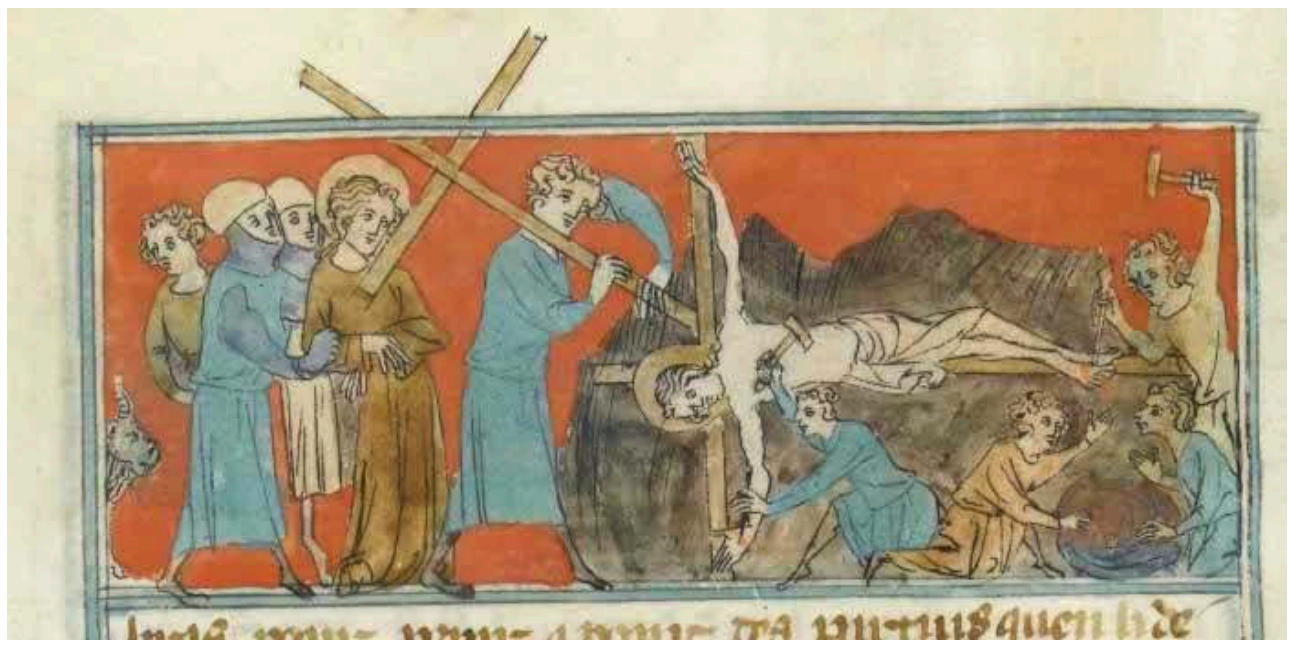

Ci nous dit, Musée Condé, ms. 26, f. 38v

Cette union intime entre le mot et la chose, cette correspondance perpétuelle, se ressent fortement lors de la lecture du texte rabelaisien, quand bien même ce grand linguiste renaissant eut parfaitement conscience de l'arbitraire du signe, lui qui jouait à merveille avec les sonorités et les incongruités logiques. Rabelais se situe à l'exact croisement du langage adamique, rêve de transparence perdue, et du langage babylonien, terreur de l'éclatement des signes et des sens. A ce carrefour, le mot animal occupe une place remarquable dans la mesure où le premier geste d'Adam consiste en la nomination des animaux. Ce geste créateur - dans l'ordre de la fiction et non dans le texte biblique - , Rabelais le répète à l'envi dans son œuvre riche de néologismes et d'inventions (au sens étymologique de « découverte, trouvaille »). Nommer, c'est créer, faire advenir l'œuvre, la fiction. Si, quelques décennies après Rabelais, dans l'Espagne de 1605 , Cervantès ouvre son grand œuvre par une scène de nomination ${ }^{5}$, le docteur en médecine ne recherche pas une telle mise en scène burlesque, ses emplois du nom animal sont disséminés à travers l'œuvre, sans nulle dimension inaugurale. Chez Rabelais, il ne s'agit pas de nommer un animal particulier, mais de rejouer en permanence la simple nomination de l'animal, dans la fraîcheur du mot brut, sans description, sans qualification, dans la verticalité d'une liste à la mesure de la diversité naturelle. Les listes d'animaux sont florissantes dans le Quart livre qui en compte trois, concentrées dans les derniers chapitres: les deux listes des offrandes à Manduce (chapitres LIX et LX), et la liste des animaux venimeux ${ }^{6}$ (chapitre LXIV). L'abondance de courtes comparaisons animales va également dans le sens de cette dissémination de l'image animale à travers l'œuvre. C'est, sans conteste, l'image la plus utilisée par Rabelais. Dans sa diversité d'emploi, l'image animale perdure en tant que figure solide, matérielle, par son évidence ou par sa résistance. La courte comparaison, non reprise en réseau, crée un effet de stupeur. Le comparant s'impose dans sa globalité, sans se confondre avec le comparé. En ce sens, on serait autorisé à comparer cette dynamique esthétique avec celle qui est à l'œuvre dans le Ci nous dit (recueil d'exempla du XIVème siècle) dans la mesure où, dans ce manuscrit, l'œil est d'abord frappé par la présence physique de l'image avant d'en pouvoir comprendre le sens, sens qui ne sera délivré que par la glose. On pourrait prendre, à titre indicatif, cette image où la tête d'un veau apparaît à l'extrême gauche d'une scène de portement de croix (fig. 1). Le veau n'a que faire dans cette scène, il faut se reporter au texte pour comprendre qu'il incarne une 
analogie christique: "et Jésus marchait derrière lui aussi docilement qu'un veau derrière le boucher " (Ci nous dit, chapitre 61). C'est cette même stupeur ${ }^{7}$ qui saisit le lecteur de Rabelais à la lecture de certaines images telles que "Dieux et Deesses s'eclaterent de rire, comme un microcosme de mouches » (Quart livre, Prologue, p. 531), « [Panurge] estoit eximé comme un haran soret » (Pantagruel, chapitre XIV, p. 263), sauf que, chez Rabelais, le phore (le comparant) conserve son opacité, il n'est jamais explicité, encore moins «moralisé ». Aussi peut-on parler dans son cas, d'une image réelle et rémanente.

4 Par cette autonomie, par cette suffisance, le mot animal paraît s'imposer avec évidence, sans recours à l'indirect de la langue conventionnelle. La langue adamique, proche de la conception cratyliste $^{8}$ du langage, fait correspondre le mot et la chose, sans rupture, si bien que le mot animal semble former une image immédiate. Cette image pourtant n'appartient pas au monde référentiel, elle est copie, simulacre. Rabelais crée l'illusion d'un mot visible, d'un texte matériel. Jean-Claude Schmitt a bien insisté sur cette qualité profonde de l'image qui ne serait pas cantonnée à un domaine, mais polyvalente, en formulant la notion d'imago:

Plus qu'un simple rapport texte/image, nous rencontrons donc un grand nombre de modalités diverses de l'imago, irréductibles les unes aux autres, mais également inséparables les unes des autres. Elles organisent le grand jeu croisé du rêve, du langage, des images matérielles. C'est ce jeu qui définit le champ complet de « l'image » médiévale, appelant à des études interdisciplinaires, plus complexes qu'on ne le pensait traditionnellement, puisqu'est mis résolument en cause l'isolement des disciplines qui s'attachaient à rendre compte séparément de chacun de ces domaines [.... ${ }^{9}$

Chez Rabelais, le lire et le voir semblent être tout autant imbriqués que le boire et le manger. Au seuil de ses livres centraux, il invite le lecteur à voir ${ }^{10}$. Une figure de construction significative réunit les prologues du Gargantua et du Tiers livre. Elle se fonde d'abord sur un hypozeuxe (reprise d'un même patron syntaxique), puis sur un chiasme (figure en forme de croix). Ces figures de ligature, au service d'un éloge de la vue, unissent irrésistiblement ces deux prologues, et invitent à les lire en diptyque. Les discours encomiastiques qui les portent sont, de manière symptomatique, associés, pour l'un, à un animal anthropomorphique, et, pour l'autre, à un homme zoomorphique : le chien philosophe de Platon, et le philosophe chien que se proclamait être Diogène. On relève de nouveau un chiasme, non plus de construction, mais de pensée. Dès lors, il s'avère nécessaire de jeter un pont entre ces deux prologues qui lient inextricablement et la lecture et la vue, et l'homme et l'animal :

Mais veistes vous onques chien rencontrant quelque os medulare? C'est comme dict Platon lib. II de rep. la beste du monde plus philosophe. Si veu l'avez: vous avez pu noter de quelle devotion il le guette : de quel soin il le guarde : de quel ferveur il le tient, de quelle prudence il l'entomme : de quelle affection il le brise : et de quelle diligence il le sugce. ${ }^{11}$

Bonnes gens, Beuveurs tresillustres, et vous goutteux tresprecieux, veistez vous oncques [constitue l'hypozeuxe du " veistes vous onques » cité ci-dessus] Diogenes le philosophe Cynic? Si l'avez vu [forme un chiasme avec le «si veu l'avez» de l'extrait précédent], vous n'aviez perdu la veue: ou je sui vrayement forissu d'intelligence, et de sens logical. C'est belle chose veoir la clairté du (vin et escuz) soleil. J'en demande à l'aveugle né tant renommé par les tressacrés bibles : lequel ayant option requerir tout ce qu'il vouldroit, par le commandement de celluy qui est tout puissant, et le dire duquel est en un moment par effect representé, rien plus ne demanda que veoir. ${ }^{12}$ 
6 Etant donné l'importance primordiale des prologues, qui offrent un vade-mecum de lecture et d'interprétation au lecteur, on pourrait considérer ces textes-pendants comme un portique signalant au lecteur le caractère foncièrement matériel de la figure verbale chez Rabelais. De fait, l'écrivain enjoint le lecteur de voir ce qu'il dénomme. Rabelais insiste tout au long de son œuvre sur cette préséance du voir dans ses chroniques. Si l'on se réfère à l'index général établi par Marie-Luce Demonet ${ }^{13}$, on remarque qu'il a utilisé pas moins de cent vingt-huit fois le verbe veoir, rien que sous sa forme infinitive. La même année 1977, Michel Charles ${ }^{14}$ et François Rigolot ${ }^{15}$ ont relevé cette propriété de l'image chez Rabelais qui semble se libérer, tout en s'y intégrant, de la figure qu'elle habite. M. Charles parle à ce propos d'une "autonomie " des descriptions dans le Prologue du Gargantua, qui sont reconnues, seulement après coup, comme des métaphores; et F. Rigolot évoque, à propos de la puce enchâssée dans l'oreille de Panurge, un phénomène de "défiguration ». Ainsi, l'image dépasse pour ainsi dire son statut littéraire, elle rejoint une certaine matérialité. Si l'on prend le terme figure, choisi par F. Rigolot, il est remarquable qu'il retrouve chez Rabelais le sens étymologique de figura : « chose façonnée ». La figure animale prend une réalité propre, une expressivité intrinsèque.

\section{L'analogie homme/animal chez Rabelais : entre continuité et rupture, le mouvement perpétuel}

7 L'analogie est une figure de similitude, de rapprochement. Nous venons de mettre en valeur l'autonomie du comparant chez Rabelais. Dès lors, l'analogie devient figure de discontinuité, d'écartèlement. Elle ne réunit pas, elle anime des images, les met en mouvement dans un cercle où comparants et comparés entrent en lutte, au lieu de s'annuler dans la restauration d'une unité perdue. En cela, l'usage de l'analogie chez Rabelais va à l'encontre d'une conception unificatrice de l'analogie, telle qu'elle a été héritée de Platon, et telle que Philippe Descola la présente dans son ouvrage Par-delà nature et culture ${ }^{16}$. P. Descola rappelle le développement de M. Foucault dans « La Prose $\mathrm{du}$ monde " à propos de l'analogie, tout en privilégiant la dimension unificatrice de celle-ci, alors qu'elle comporte nettement deux aspects: convenientia et aemulatio. Conçue comme engendrant des effets de corrélation, l'analogie selon P. Descola instaure un système statique d'équivalence terme à terme. La nouvelle identité créée constitue le résultat de la fonte des objets comparés. Aussi le devenir analogique s'arrête-t-il au moment de la constitution d'une nouvelle correspondance. M. Foucault, quant à lui, pense un système beaucoup plus instable qui met en équivalence non des termes, mais des rapports. Il insiste sur la lutte, l'aemulatio, qui anime les entités mises en rapport. Aucune ne s'annule dans la constitution d'un paradigme commun. Toutes demeurent dans leur singularité tout en étant intégrées, par le processus analogique, dans le mouvement global qui parcourt les êtres du monde, à savoir ceux de sympathie et d'antipathie. L'analogie participe alors au flux du devenir, se situe entre le lien et l'affrontement. C'est à cette espèce qu'appartient l'analogie rabelaisienne. En ce sens, Rabelais s'inscrit en faux vis-à-vis de l'analogisme médiéval, pensé comme un moteur d'unification de la Création. Il se démarque également d'une certaine épistémè renaissante qui tente de rassembler la diversité du monde pour mieux l'appréhender. L'esthétique de ses chroniques met en crise l'esprit encyclopédique au moment même où elle l'intègre et en fait sa matière. Chez lui, l'analogie fait trembler l'image, sème le 
trouble ontologique; le phore (le comparant) et le thème (le comparé) deviennent parfois indistincts. C'est le cas de la grande analogie inaugurale du Gargantua, qui rappelle la comparaison de Socrate aux silènes ${ }^{17}$. Le comparé (Socrate) encadre la structure, mais celle-ci s'étend et se multiplie de telle sorte que les comparants acquièrent une autonomie indéniable. Cette chaîne analogique prend beaucoup d'ampleur si bien que chaque maillon recouvre son indépendance. En voici la structure :

\begin{tabular}{|l|l|l|}
\hline Comparés & $\begin{array}{l}\text { Outils de } \\
\text { comparaison }\end{array}$ & Comparants \\
\hline $\begin{array}{l}\text { Socrates prince des } \\
\text { philosophes }\end{array}$ & semblable es & Silenes \\
\hline $\begin{array}{l}\text { Silenes estoient jadis } \\
\text { petites boites }\end{array}$ & telles que & voyons de present es boutiques de apothecaires \\
\hline \hline & & figures joyeuses et frivoles \\
\cline { 2 - 3 } & & $\begin{array}{l}\text { comme de Harpies, Satyres, oysons bridez, lievres cornuz, } \\
\text { canes bastées, boucqs volans, cerfz limonniers, }\end{array}$ \\
\hline Silene maistre du bon & Quel [fut] & Et aultres telles pinctures contrefaictes à plaisir pour \\
Bacchus & exciter le monde à rire \\
\hline Socrates & & \\
\hline
\end{tabular}

8 La symétrie est parfaite entre les deux thèmes Socrate et Silène, tandis que les phores s'épanouissent dans l'interstice ouvert par cette structure analogique. Une correspondance notable s'établit entre les «figures joyeuses et frivoles» et les "peintures contrefaites à plaisir pour exciter le monde à rire ». L'énumération encadrée par ces deux mentions picturales s'ouvre par des figures traditionnelles de l'iconographie antique : les Harpyes et les Satyres. Ainsi, à l'orée de cette accumulation, se trouvent des êtres hybrides, la Harpye ${ }^{18}$ au visage de femme et au corps d'oiseau, et le Satyre (assimilé au silène) dont le bas du corps correspond à un cheval ou à un bouc $^{19}$. A la suite de ces figures attendues, appelées par la mention de Silène, viennent des animaux tout différents, plutôt domestiques, malgré quelques attelages étonnants, tels que les oisons bridés, les canes bâtées (le jeu de mots se fait sur le syntagme " âne bâté »), et les cerfs limoniers (Rabelais transforme les domestiques bœuf ou cheval limoniers en un image paradoxale transformant un animal sauvage, le cerf, en un animal de trait). Cet incongru mélange d'hybrides mythologiques, d'animaux domestiques ou sauvages, et d'animaux fantastiques issus d'un bestiaire plus populaire (les lièvres cornus et les boucs volants), rompt les rapports de similitude, et confère à cette énumération de comparants une liberté croissante. Bride, bât, limon : les animaux 
domestiques - on domestiqués par les jeux linguistiques de l'auteur - sont caractérisés par une retenue, une maîtrise, tandis que Satyre et Harpye sont caractérisés par leur liberté et leur excès. Aussi cette juxtaposition d'animaux a-t-elle une certaine logique antithétique. Toutefois, il est évident que Rabelais recherche avant tout le surgissement de l'image insolite, afin d'enrayer toute possibilité de reconnaissance chez le lecteur. Il le confronte, selon son habitude, à un imaginaire fondé sur la rupture et la discontinuité, afin de dégager nettement des images hors jeu, qui ne se soumettent pas à l'analogie. Ainsi, fourmillent autour de Socrate des images animales irréductibles à sa personne, grâce au détour fécond par les boîtes peintes de figures grotesques et d'animaux familiers. En dernière instance, resurgit une métaphore animale appartenant au domaine domestique : le regard de taureau, traditionnellement attribué et à Silène et à Socrate. Entre détachement et rattachement, les images animales travaillent à approfondir, dans leur superficialité paradoxale de «figures joyeuses et frivoles ", le sens d'une humanité d'autant plus sage et établie qu'elle vacille sur les eaux troubles de son animalité. En cela, les figures tutélaires de Socrate et de Diogène sont particulièrement éloquentes, puisque ces deux grands penseurs de l'humanité se situent volontairement au seuil de l'animalité, et font, joyeusement, figure de bêtes.

\section{Exubérance et délitement analogiques : le sens introuvable}

9 Une question demeure : quel est le sens de ce bestiaire omniprésent, exubérant, sans cesse à la marge de la narration ? C'est la question que s'est posée Michael Camille au seuil de son livre Images dans les marges, à propos des images marginales des siècles gothiques (tant dans les manuscrits que dans la statuaire):

Je pourrais commencer par me demander, à la manière de saint Bernard, ce que signifient tous ces singes lascifs, ces dragons qui se dévorent eux-mêmes, ces têtes pansues, ces ânes jouant de la lyre, ces prêtres baise-culs et ces jongleurs acrobates qui surgissent dans les marges des édifices, des sculptures et des manuscrits enluminés du Moyen Âge. Mais je m'intéresse davantage à la façon dont tout ce monde prétend éluder le sens, comme pour célébrer non pas « l'être » mais, bien plutôt, le flux du « devenir » $[. ..] »^{20}$

10 L'élément imageant de l'analogie correspondrait chez Rabelais au lieu de la marge. Toujours décrochée, l'image animale introduirait, au cœur de la narration, un espace de jeu, permettant la redéfinition perpétuelle de l'homme. Toujours inquiétée, toujours mise à mal, la notion d'humanitas n'en devient que plus forte. Au contraire de son maitre Erasme qui récuse toute forme d'animalité dans son Traité de civilité puérile ${ }^{21}$, Rabelais allonge avec plaisir les enfances malpropres et bestiales de Gargantua. Au-delà du jeu intertextuel, émerge une conception singulière de l'humanité qui se situe bien plutôt dans "le flux du devenir » évoqué par M. Camille que dans un quelconque figement identitaire. Cette tension vers une altérité menaçante alimente le corps des images rabelaisiennes où, sans cesse, l'homme se voit comparé à l'animal. Le Quart livre comporte une description saisissante de ce point de vue-là. Il s'agit de l'anatomie de Quaresmeprenant, sorte de monstre allégorique au nom antiphrastique: il incarne toutes les contritions hypocrites des partisans du carême alors que son nom dénote la période des trois jours gras qui précède ce temps de jeûne. Cette anatomie, loin d'offrir une vision claire, explicative de l'être décrit, présente un fourmillement d'images antagonistes, prises dans un réseau analogique d'une étendue effrayante. C'est 
Xenomanes qui s'attelle à la description de Quaresmeprenant, description d'un être absent, que Pantagruel ne voit pas, mais que son compagnon lui donne à voir. L'absence physique de Quaresmeprenant conditionne l'absence de naturel de la description, mime verbal de la figure contre-nature qu'est Quaresmeprenant. La situation d'énonciation, au large de l'île de Tapinois, permet un plan large sur la vérité de cet individu. La proximité de l'hypocrisie eût provoqué l'adhésion aveugle, la prise de distance s'est imposée. Cette distance est comprise comme distance spatiale et distance critique. Xenomanes, le savant de l'équipée, adopte le langage du médecin en procédant à l'« anatomie » de Quaresmeprenant. Il n'en fait pas le portrait, mais l'anatomie, car un portrait aurait manqué le dessous du personnage hypocrite. Aussi la description de Xénomanes est-elle doublement descriptive, elle décrit ce qui est, et ce qui est caché. C'est une sorte d'ultra description, étalant tout Quaresmeprenant au grand jour du logos. Pantagruel dit à Xenomanes «[...] aussi me exposez sa forme et corpulence en toutes ses parties $\aleph^{22}$. La réalité ne montre pas la vérité d'une âme qui peut toujours se déguiser sous des masques. Cette description se présente comme une désagrégation de l'unité du vivant et même comme une annulation du vivant, comme une mortification par la prolifération. Terence Cave dans sa Cornucopia voit en Quaresmeprenant l'incarnation de l'abios bios évoquée dans le Prologue: «[...] sa stature colossale est littéralement abiotique en ce qu'elle prolifère et croît selon un processus négatif, désagrégateur, qui s'éloigne progressivement de toute donnée d'expérience ${ }^{23}$. L'anatomie de Quaresmeprenant prend des allures d'atomisation analogique. Toutes ses parties, tant internes qu'externes, et toutes ses contenances sont explorées et mises en rapport avec des éléments étrangers et similaires. Ces similitudes fonctionnent sur des rapports bizarres et surprenants, ayant trait à des référents très différents. Cela crée une dynamique contraire à l'effet attendu par le procédé analogique, une dynamique non pas centripète, mais centrifuge. Le petit monde qu'est le corps vivant s'évanouit en une explosion verbale :

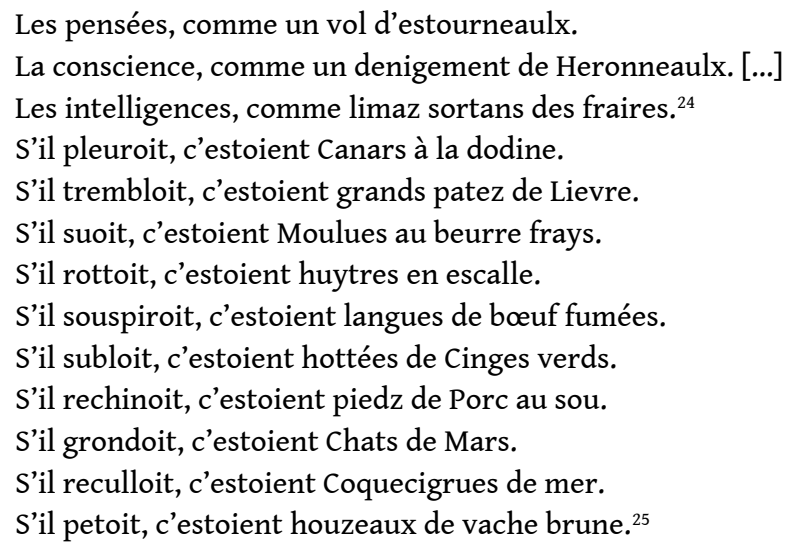

11 Rabelais pousse à l'extrême les tendances analogiques des textes anatomiques sérieux. $\mathrm{Au}$ nom du comique et de la satire, il instaure des analogies pour le moins surprenantes. Dans l'anatomie des «parties internes» du monstre, le mouvement de " perte » s'illustre par des images de fuite d'animaux - rien ne demeure en ce corps qui n'est que dispersion et évanouissement, tout en étant la source d'un extraordinaire foisonnement verbal et imaginaire. L'éparpillement des pensées de Quaresmeprenant (l'écart entre ses intentions officielles et ses officieuses) est figurée par la comparaison avec un vol d'étourneaux, la diversité fallacieuse de sa conscience par un envol de héronneaux, la lenteur de son intelligence par des limaces sortant de fraises. En faisant le rapport des « contenences ${ }^{26}$ » de Quaresmeprenant, Rabelais transforme les matières 
mortes en un texte animé. Il choisit d'utiliser des verbes conjugués au lieu des noms pour faire référence à celles-ci, et de leur accoler des références animales. La figure analogique de Quaresmeprenant fonctionne par une duplication permanente. A chaque fois, l'image ajoutée à l'élément comparé s'en éloigne considérablement. L'anatomie de Quaresmeprenant se multiplie en écartèlements analogiques. Toutes les ressemblances donnent à voir un individu en déliquescence, se perdant infiniment dans une myriade de comparants qui sont autant d'éloignements de l'unité naturelle. A la lecture de ces anatomies le lecteur ne peut pas se représenter cet être. Il visualise successivement des réalités hétéroclites. Ainsi le processus analogique est-il détourné de sa fonction naturelle qui est de rassembler la diversité du réel pour lui donner un sens. Ce dévoiement de l'analogie mime la figure contre-nature qu'est Quaresmeprenant. La «rhétorique à la dérive ${ }^{27}$ » de Rabelais imite l'être dénaturé qu'elle prend pour objet. Quaresmeprenant vit dans un monde inversé. Il est une antithèse de Nature, un simulacre malin de celle-ci :

Peschoit en l'air, et y prenoit escrevisses decumanes. Chassoit on profond de la terre, et y trouvoit Ibices, Stamboucqs, et Chamoys. ${ }^{28}$

12 La thématique du monde inversé s'insère naturellement dans cette «description inversée ». Tentative d'épuisement des facettes de Quaresmeprenant, cette énumération ne délimite pas son être, ne le construit pas, ne nous le fait pas connaitre. Elle est ouverture infinie, pullulement vertigineux, sape du processus de reconnaissance. La prodigalité analogique fait perdre de vue l'objet de ces comparaisons. Les anatomies de Quaresmeprenant outrepassent l'ordre naturel du discours, transgressent les règles de l'art. Finalement, toutes ces discordances, toutes ces cacophonies, servent une seule et même intention, celle de détruire par éclatement, par confusion, la cible de cette satire : les idéologues. Rabelais épouse de son écriture Antiphysie, pour enfanter un nouvel Amodunt: Quaresmeprenant, incarnation monstrueuse de toutes les espèces d'idéologues fanatiques. Pantagruel, à la fin de l'épisode de l'île de Tapinois, raconte une histoire lue parmi « les Apologues antiques ", celle de Physis et d'Antiphysie. Antiphysie, jalouse des enfants de Physie, Beauté et Harmonie, enfanta Amodunt (démesure) et Discordance. De cette généalogie mythique, Rabelais tire l'origine poétique de ses ennemis :

Depuys elle engendra les Matagotz, Cagotz, Papelars: les Maniacles Pistolez: les Demoniacles Calvins imposteurs de Genève: les enraigés Putherbes, Briffaulx, Caphars, Chattemites, Canibales : et aultres monstres difformes et contrefaicts en despit de Nature. ${ }^{29}$

13 Rabelais extrait de la variété et de l'abondance de la nature des images qu'il réinvestit dans une figure composite, fruit du dévoiement de la fécondité naturelle. La corne d'abondance naturelle se mue en corne stérile, image de la mauvaise foi dévastatrice des idéologues. Les idéologues de tous bords sont attaqués : le réformé Calvin qui s'en était pris nommément à Rabelais dans son ouvrage Des Scandales (Genève, 1550), et le religieux de l'abbaye de Fontevraud, docteur à la faculté de Paris, Gabriel de PuyHerbault qui avait fait, dans son Theotimus (1549), un portrait épouvantable de l'auteur de la geste pantagruélique. L'ecclésiastique y présentait un Rabelais « imitant le singe à la longue queue ", souillant « son papier d'infamies », vomissant « un poison qui infecte peu à peu toutes les contrées". Au portrait-charge traditionnel, Rabelais oppose sa créativité et son audace. Il répond à ces attaques par un extraordinaire renouvellement des procédés du bestiaire satirique. 
14 Ainsi, Rabelais "élude le sens" par des images volontairement marginales, qui décontenancent, annulent toute forme de reconnaissance aisée. Cette figure innommable, irreprésentable, cet hybride outré, fait triompher la force de l'imagination ${ }^{30}$, après avoir fait admettre l'échec de la représentation. En perpétuel devenir, l'image animale ne se fixe à aucun moment en une identité stable, elle glisse, échappe, tandis que son créateur figure, défigure, multiplie les formes, informe, déforme, réforme, afin de mieux imposer la difficile saisie de l'homme, d'autant plus lorsqu'il s'agit d'exprimer sa dimension malfaisante - comme ce fut le cas ici.

\section{Anti-nomination et figuration : de l'inversion du signe à sa matérialité}

15 On retrouve dans le Quart livre un rapport similaire à la figuration, à savoir un renversement étudié des procédés de représentation. Au chapitre LXIV, la liste des animaux venimeux par son caractère illisible, incompréhensible, offre une image rigoureuse du caractère indicible du mal. La liste en elle-même devient la forge d'un monstre verbal qui tire sa force évocatrice de son défaut de signification. Cette accumulation de noms d'animaux se caractérise en effet par une multiplication de xénismes (noms étrangers, non intégrés dans la langue qui les transcrit). Ces noms communs se réduisent proprement à leur graphie ou à leur sonorité, c'est-à-dire à leur pure matérialité. Aussi Rabelais renverse-t-il ici la nomination adamique, puisque ces désignations éloignent leur référent, l'annulent même, au lieu de le rapprocher, de le circonscrire, au lieu de signer une appartenance réciproque entre le mot et la chose. Rabelais opère bien alors ce travail d'« échouage » du langage dont parlent les auteurs d'Image et transgression au Moyen Âge :

A signifier une réalité monstrueuse, physique autant que métaphysique, le nom échoue: nommer, c'est rabattre le sens. La représentation échoue aussi: représenter, c'est entraver l'esprit. La réalité, conçue et éprouvée comme innommable et irreprésentable, excède l'imagination. Il faut alors «échouer » le langage et la représentation pour exprimer le transgressif. Ce qui transgresse vraiment passe l'ordre et la mesure de la conscience. Ceci n'a pas échappé à ceux qui prenaient la parole, la plume ou le pinceau : ainsi, la «bête » n'avait-elle pas d'image et l'hybride n'avait-il pas de nom. A l'inverse, en «nommant» visuellement, la stricte représentation matérielle fermait la perception du monstrueux et entravait le travail de l'imagination: nommé et reconnu entre mille, l'animal fantastique (licorne, griffon, chimère, manticore) ne représentait aucun danger qui ne fût désamorcé, aucune réalité qui ne fût déjà objectivée. ${ }^{31}$

Il semble en effet que l'auteur renaissant se situe très précisément dans ce type de rapport au nom animal, et même qu'il soit hautement conscient de ce procédé d'appropriation de l'animal par sa nomination. Dans sa fiction, la licorne devient un animal parfaitement domestique dans la mesure où elle ressortit totalement à la culture livresque classique. La licorne, dans cette lettre de Pantagruel à son père, devient plus domestique et apprivoisée qu'un chaton :

Je vois envoie pareillement troys jeunes Unicornes plus domesticques et apprivoisées, que ne seroient petitz chattons. J'ay conféré avecques l'escuyer, et dict la manière de les traiter. Elles ne pasturent en terre, obstant leur longue corne on front. Force est que pasture elles prenent es arbres fruictiers, ou en ratelliers idoines, ou en main leur offrant herbes, gerbes, pommes, poyres, orge, touzelle : brief toutes espèces de fruictz et de legumaiges. Je m'esbahis comment nos 
escrivains antiques les trouvent tant farouches, feroces, et dangereuses, et oncques vives n'avoir esté veues. Si bon vous semble ferez épreuve du contraire: et trouverez qu'en elles consiste une mignotize la plus grande du monde, pourveu que malicieusement on ne les offense. ${ }^{32}$

17 Au contraire, la liste du chapitre LXIV impose au lecteur un effet de totale étrangeté, de totale méconnaissance. Eusthènes rompt une conversation qui, même si elle était cacophonique et très peu logique, était commune. Dans le même temps, Rabelais met à mal les principes énoncés dans la dédicace au seigneur Odet, cardinal de Châtillon, en alimentant une liste hermétique - véritable gangrène du corps signifiant - à la fin de son roman. La discordance entre cette dédicace et le contenu du chapitre LXIV est flagrante: d'un côté, bonne santé et bonne vinée, de l'autre, malaise et venin. Dans cette dédicace, Rabelais développe le thème de l'influence du visage du médecin sur le patient. Il semble qu'il faille tirer de ce développement une analogie avec l'écriture et la lecture. L'écriture elle-même doit avoir «la face joyeuse, sereine, gratieuse, ouverte, plaisante ${ }^{33}$ » afin de réjouir le lecteur. Or, quel plaisir le lecteur peut-il tirer de ce catalogue qui prend des allures de catastrophe érudite? Le lecteur lit, tout au plus, les premiers mots et les derniers du catalogue. Roland Barthes, dans sa Leçon ${ }^{34}$, insiste sur l'importance de l'«indirect» en littérature. L'homme de lettres ne peut, selon lui, retranscrire directement dans sa prose un savoir scientifique. Il lui faut observer un détour salvateur. Or, Rabelais impose une liste herpétologique qui se veut exhaustive. Sommes-nous toujours en littérature ? Où est l'« indirect » si "précieux »? Où se trouve le travail de la langue littéraire ? Peut-être au sein même de cette impertinence, de cette incongruité : placer en fin de chapitre, et trois chapitres avant la " conclusion » du roman, une masse incompréhensible. Cette figure opaque tient forcément un rôle allégorique. En attendant le dévoilement, demeurent la surprise, l'étonnement, l'incompréhension. L'effet sur le lecteur est incontestablement puissant, à la mesure de cette figure démesurée - sangsue gorgée de venin et d'obscurité sémantique. Cette liste fait matériellement, visiblement figure, elle s'impose avant tout par la typographie, la graphie et les sonorités. On en reste à cette épaisseur sensible du signe, qui obstrue l'accès au sens pour mieux révéler une infamie, mieux traduire l'horreur de la persécution idéologique. Cette figure introduite par une prétérition « pour lors seront loin de moi", dans sa sur-présence physique (on compte quatre-vingt-dix-huit appellations), désigne une absence. Voit-on autre chose à cette lecture qu'un trou noir, qu'un " abysme de science ${ }^{35}$ " ? Si le roman doit donner à voir, une scène, une idée, ici son rôle se dérobe. Pourtant, cette dérobade porte en elle-même son propre sens. Ce monticule venimeux, s'il fait l'admiration des linguistes et des lexicographes, contient une charge négative, destructrice. Le contenant - la liste démesurée - et le contenu des noms d'animaux venimeux aux sonorités imprononçables et incompréhensibles sont en parfait accord et se renforcent l'un l'autre pour envenimer le texte :

Aspics.

Amphisbenes.

Anerudutes.

Abedissimons.

Alhartafz.

Ammobates.

Apimaos.

Alhatrabans.

Aractes.

Asterions.

Alcharates. 
Arges.

Araines.

Ascalabes.

Attelabes.

Ascalabotes.

Aemorrhoides.

[...]

Tarantoles.

Typholopes.

Tetragnaties.

Teristales.

Viperes. ${ }^{36}$

Fig. 2

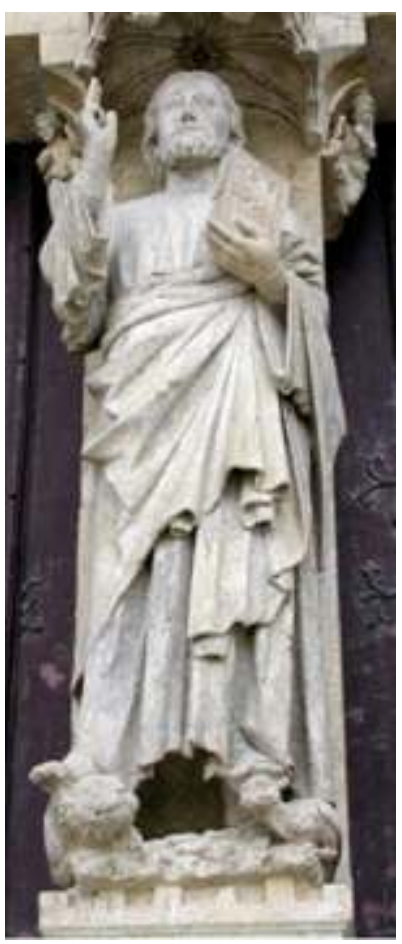

«Beau Dieu », Cathédrale d'Amiens, v. 1220-1230

Dans le monde animal, Rabelais a trouvé une mine de représentations débordantes de possibilités de variation et extrêmement riches de suggestivité. Son imagination est incontestablement marquée par ce champ de figuration. Le plus remarquable ne réside pas tant dans l'omniprésence de ces images que dans l'audace de leur emploi. Rabelais écrase avec la même force que le "beau Dieu " d'Amiens ${ }^{37}$ (fig. 2) les ennemis de sa religion et de sa littérature, mais il n'écrase pas un aspic et un basilic, symboles reconnaissables, il enserre tous les animaux venimeux. Si cette liste d'animaux fait symbole, si elle crée un lien analogique avec une réalité cryptée - celle de l'hypocrisie, il s'agit d'un processus analogique singulier, quasi inversé. La face matérielle du symbole doit faire signe vers une autre dimension, vers une signification spirituelle ou allégorique $^{38}$. Or, ici, le signe en soi n'est pas connaissable, n'est pas abordable. Le signe lui-même se refuse. Toutefois, cette opacité du verbe traduit, à l'échelle macrostructurale de la liste, une signification très forte : elle offre de manière sensible la négativité du mal tel que le pense Rabelais. Le «terrorisme» des hypocrites et des 
idéologues perce à travers cette trace sensible de l'excès maléfique. Le mal excède la représentation. Seule cette anti-nomination pouvait figurer ce qui outrepasse les moyens ordinaires du langage et de la représentation. Dès lors, le texte devient animal venimeux, et la liste, venin purement signifiant et sensible, devient l'innommable, le détracteur, le persécuteur.

\section{Une figure marginale : Panurge « eschappé es chiens $^{39}$ »}

19 Le personnage de Panurge est particulièrement intéressant concernant cette analogie entre l'homme et la bête. Il est, par excellence, l'homme qui brouille les frontières, menace la stabilité des catégories. Il fait advenir l'analogie sur le plan narratif. Son apparition dans le livre est frappée du signe canin ${ }^{40}$, sceau qui ne le quittera plus :

Ung jour Pantagruel se pourmenant hors de la ville, vers l'abbaye Sainct Antoine, devisant et philosophant avecques ses gens et aulcuns escolliers, rencontra ung $\underline{\text { homme, }}$ beau de stature et élégant en tout linéamens du corps, mais pitoyablement navré en divers lieux, et tant mal en ordre qu'il sembloit eschappé ès chiens, ou mieulx ressembloit à ung cueilleur de pommes du pays du Perche. ${ }^{41}$

Les premières informations que nous recevons sur Panurge forment un portrait inaugural très instructif. D'abord, on le rencontre "hors de la ville", indication spatiale significative qui dit sa marginalité. Cette situation hors de la ville exclut Panurge de tout pacte d'urbanitas. Il n'a aucun engagement vis-à-vis de la cité, puisqu'il est issu d'une sorte de no man's land, soustrait à toute loi. L'origine obscure de Panurge, son irréductible étrangeté ouvre le lieu commun de l'homme déraciné, insouciant et amoral. Il se place hors des remparts (remparts qu'il tourne allègrement en dérision au chapitre XI), et se détermine d'emblée par son rapport à la transgression, au-delà de la ville et au-delà de la civilisation. Il expose l'image paradoxale de sa beauté et de sa misère. A la fois beau et blessé, « navré », il semble échappé des griffes et des dents des chiens. Il est marqué dans sa chair par la férocité canine. Et son comportement restera lié à cette ascendance, comportement que l'on pourrait assimiler à l'attitude cynique qui ne se soucie ni de la morale, ni des bonnes mœurs. Le cynisme, issu du grec kunismos tire son nom du chien kuôn; l'homme cynique adopte en effet le mode vie "d'un chien ", dans la mesure où il ne se plie à aucune règle sociale, et vit proprement en hors la loi. Il vit dans la rue, en misérable, parce qu'il est lucide sur la condition misérable de l'homme. Nous ne dirions pas que Panurge adopte une attitude de philosophe cynique. Il est cynique par accident, sans aucune conviction. Son cynisme relève plutôt du sens péjoratif qu'on lui donne depuis le XIXème siècle : brutalité et impudence. Cependant, ce « cynique » n'est pas repoussant, au contraire, il est aimable: «Comment Pantagruel trouva Panurge lequel il ayma toute sa vie». Si Pantagruel est immédiatement séduit par la beauté naturelle de Panurge, c'est que celui-ci respire la "grâce animale ${ }^{42}$ »; son corps laisse paraître une négligence telle qu'il émane de lui une sorte d'inconscience ou de naïveté qui le fait paraître tout droit sorti d'une meute de chiens ou d'un verger du Perche. Ce portrait inaugural est imprégné de fascination, Pantagruel est littéralement sous le charme de cet homme énigmatique, sorti de nulle part. On lit la curiosité du géant dans la série de questions empressées qu'il lui lance :

Qui estes vous? Dont venez vous? Où allez vous? Que quérez vous? Et quel est vostre nom $?^{43}$ 
21 Panurge est le lieu parfait de l'ambiguïté propice à toutes les perversions. Il va s'ingénier à toutes les corruptions de la nature humaine. Il entreprend, au chapitre XIV, de «venir au dessus d'une des grandes dames de Paris» (p. 116). Avant qu'il ne fasse appel aux chiens, Panurge utilise un vocabulaire animal pour parler des rapports humains "que feussiez couverte de ma race» (p. 116). Le but de Panurge est déjà perceptible, il veut dépasser la «haulte dame de Paris», la faire déchoir pour triompher. La séduction ne semble pas l'intéresser, si ce n'est en tant que jeu immédiatement détruit par la dérision. Ce qui l'intéresse, c'est la perdition. Son triomphe consistera dans l'humiliation de cette " haulte dame » et le rire triomphant de lui-même. Ce maniaque des mariages contre nature (mariage des vieilles, mariage des vesnes et des vesses, mariage du roi Anarche à une bonne vieille) décide d'unir cette femme à tous les chiens de la ville. Il lui impose l'animalisation en imprégnant les plis de sa robe de l'odeur d'une chienne en chaleur. Toute la composition du texte concourt à l'assimilation de la femme à la chienne. Les chiens sont pratiquement toujours au pluriel, ils forment une meute indéfinie, impossible à identifier, alors que la chienne est singulière (sa mise à mort et son excision sont longuement décrites), les deux « femelles » se confondent aisément dans l'économie du texte :

Je croys que ceste dame là est en chaleur, ou bien que quelque lévrier la couverte fraischement. ${ }^{44}$

Et, quant il vit que tous les chiens grondoient bien à l'entour de elle comme ilz font autour d'une chienne chaulde $[. . .]^{45}$

De plus, dans la version définitive, Rabelais choisit de citer nommément une chienne qu'il tire de la troisième Bucolique de Virgile ${ }^{46}$ :

Ce jour de la vigile, Panurge chercha tant d'ung cousté et d'aultre, qu'il trouva une chienne qui estoit en chaleur $[. . .]^{47}$

Le jour de la vigile Panurge chercha tant d'ung cousté et d'aultre qu'il trouva une lycisque orgoose $[. . .]^{48}$

23 Cette allusion à la Lycisca des Bucoliques, associée au mot d'origine grecque " orgoose » dont la racine signifie "être en rut ", donne une identité particulière à cette chienne qui prend une réalité culturelle non négligeable. Cette nomination, pour être comique dans ses sonorités et par son pédantisme (Rabelais parle ici un peu comme l'écolier limousin), n'en renforce pas moins la possible personnification de cette chienne. Cette analogie entre la femme et la chienne fait partie de l'imaginaire misogyne de l'époque. André Tiraqueau, juriste et ami de Rabelais, a écrit un traité important sur le mariage, dont certains passages montrent la force de cette assimilation :

Sed et hanc mulierum impudentiam adstruit, quod infra dicemus, cum de invidia foeminarum differemus, mulieres canibus a multis comparatas, quia eo animali nihil est impudentius, [...] De qua re loquitur Diuus Hieronimus in Esaiam, c. 66. Pulchre (ait) canis et meretrix copulantur, quia utrunque animal pronus est ad libidinem. ${ }^{49}$

24 Panurge applique à la lettre la leçon moraliste du juriste qui brocarde les femmes impudentes en les comparant honteusement à des chiens. La bestialité, au sens de rapport contre-nature entre un homme et un animal, est clairement évoquée. La scène finale du chapitre XIV constitue une sorte d'amplification effrayante de cette bestialité. Le désordre et l'avidité de cette masse de chiens accumulés sur cette femme, véritable point de mire de leur appétit sexuel, constitue une image très puissante de souillure. La femme est assimilée à la chienne par tout un travail de personnification, mais sa condition est encore pire que celle de la chienne. L'événement est absolument irréel 
par le nombre incalculable et toujours grandissant des chiens qui assaillent cette seule femme. Il s'agit, par une hyperbole numérale déréalisant ce spectacle, de figurer la situation précaire de l'homme (par-delà la dimension misogyne de la scène) qui peut à tout moment déchoir, basculer. En ce sens, l'épithète « haute » présente dans le titre ( "Comment Panurge feut amoureux d'une haulte dame de Paris ») est particulièrement parlante. Cette scène obscène, à la tonalité proche des fabliaux érotiques du Moyen Âge, narre, dans le registre grotesque, l'histoire d'une chute, et répète, de manière sous-jacente et bouffonne, le drame eschatologique de la Chute qui est marqué par la dissemblance ${ }^{50}$. Ici, la dissemblance est brutalement rappelée par une scène de bestialité.

Panurge établit une situation extrêmement transgressive. Non seulement il amène sur la scène publique un acte sexuel, mais en plus ce dernier est absolument contre nature : il scelle l'union de deux espèces différentes et celui de la multitude avec le singulier. A. Tiraqueau nous rappelle que le chien a été interdit dans la citadelle d'Athènes parce qu'il s'unissait en public et n'importe où : « quod in propatulo et passim coeat ${ }^{51}$ » . Or, Panurge introduit cette scène obscène dans des lieux sacrés : dans l'église, puis au beau milieu de la procession, et ce pendant la fête même du Corps Dieu. Il exacerbe la transgression et s'arrange pour en faire " un déduyt » (divertissement) bien visible aux yeux de tous. Cette accumulation de violations des interdits naturels et religieux fait de cette scène le paroxysme du caractère " marginal » de Panurge qui ne respecte aucune loi. Il impose sa propre vision du monde et pour ce faire utilise à merveille la dimension effrayante du mélange de l'homme à l'animal. Cet accouplement contre nature, guidé par le stratagème de Panurge, corrobore l'image d'un Panurge en retrait, qui refond les réalités, bouscule l'ordre des choses, pour le plaisir et la satisfaction d'un beau spectacle : «A quoy volontiers consentit Pantagruel, et veit le mystère, qu'il trouva fort beau et nouveau » (p. 124). L'image transgressive investit ici le cœur de l'ordre, le cœur de la norme : l'ordre religieux (l'église et la procession de la Fête Dieu), l'ordre social (l'assentiment du suzerain Pantagruel) et l'ordre moral (la femme mariée et pratiquante). Ainsi, la figure marginale de Panurge déborde son cadre naturel, dévore tout l'espace de la représentation, met à mal toute hiérarchie des catégories. Il ne s'agit pas ici d'un "montage normatif ${ }^{52}$ " comme on le rencontre dans les manuscrits gothiques où l'image centrale et le texte imposent la norme, tandis que la marge offre un contrepoint qui vient renforcer cette organisation nettement établie. Cet univers rappelle l'univers anomal du Roman de Renart ${ }^{53}$. De fait, Panurge s'inscrit bien dans la filiation d'un type : celui du décepteur ${ }^{54}$. Seulement le texte rabelaisien est autrement plus complexe que celui des branches renardiennes ou des fabliaux érotiques du Moyen Âge, dans la mesure où aucune homogénéité, aucune continuité n'est jamais atteinte. Le texte sauvegarde à tout moment son aspect de centon ${ }^{55}$. C'est un texte tissé de morceaux hétérogènes, venant de sources aussi variées que le permet l'expérience intellectuelle, sociale, professionnelle et esthétique de son auteur. Aussi le personnage de Panurge ne peut-il pas être réduit à l'archétype du décepteur. S'il en est l'héritier, il se singularise dans la mesure où il habite un univers romanesque hétérogène, mêlant érudition et rire, sérieux et grotesque, engagement et insouciance affichée. Cette figure marginale est autrement plus dangereuse que celle des Trubert et des Renart en ce sens qu'elle vient troubler un monde de savoir et de pouvoir. L'optimisme fondateur du premier roman n'est pas si évident ; une réflexion sur la légitimité du savoir humain, de la connaissance humaine, fondée sur le précepte évangéliste d'humilité, est déjà engagée. Dès le Pantagruel, Rabelais suit la voie frayée par Corneille Agrippa ${ }^{56}$, à savoir 
la remise en cause de l'habilité de l'homme à maittriser la Création. Ce point de vue déplace le rôle de Panurge, lui offre une dimension allégorique très forte: toutes les destructions, toutes les confusions qu'il sème sont autant de signes de la mise en crise de la notion d'humanitas - prétendument portée triomphalement par ce grand humaniste. Dès lors, tous les vacillements de la catégorie humaine introduits par Panurge sont autant de traces d'une réflexion fondamentale sur les droits et les pouvoirs de l'homme. Ainsi, les images de devenir animal, les images de glissement analogique, d'hybridité, sont souvent le signe grotesque d'une réflexion anthropologique cryptée.

26 Si l'analogisme est présenté par Philippe Descola comme un « rêve herméneutique de complétude", il s'abîme chez Rabelais en une dynamique agonistique paradoxale. L'auteur renaissant instaure un système analogique qui repose sur le principe d'une inclusion laconique de la figure, si bien que les images animales suscitent un sentiment de stupeur. Au lieu de servir une signification, ces images se caractérisent par leur appel à la sensibilité. Selon l'ordre esthétique rabelaisien, le sensible est premier, le sens second et le plus souvent dissimulé, travesti. Grâce à une esthétique de la surprise fondée sur l'insolite et l'incongru, l'auteur parvient à faire "surgir " l'image hors du cadre narratif, à lui conférer une épaisseur sensible. Cette matérialité de la langue, cette "incarnation" des images, est la condition nécessaire à la formation d'une pensée analogique paradoxale qui, par le rapprochement des signes, crée une tension identitaire. La co-présence maintenue des images animales et de la figure humaine (qu'elle soit explicite ou sous-jacente) offre une vision foncièrement labile de l'homme. Sujet au changement et à l'impermanence des choses, l'homme de Rabelais s'incarne sous la menace salvatrice du "devenir animal». Sans cette inquiétude, sans cette remise en jeu perpétuelle, il retomberait dans une torpeur bestiale. Les effets de juxtaposition insolite (les Harpyes, Satyres, oisons bridés, lièvres cornus, canes bâtées, boucs volants, cerfs limoniers du Prologue de Gargantua), d'écartèlement analogique (les attributs hétérogènes et faramineux de Quaresmeprenant), d'opacité du sens (les animaux venimeux introuvables du Quart livre), de violence transgressive (les outrages bestiaux fomentés par Panurge), servent un seul et même but: rapprocher l'homme et la bête, faire trembler les catégories, tout en préservant la force évocatrice des images animales, pour mieux interroger la "nature» de l'homme, homme qui n'existe que plongé dans le monde, confronté à la diversité et à la confusion d'après la Chute et d'après Babel. Selon Pic de la Mirandole, Dieu aurait dit au premier homme :

Nous ne t'avons fait céleste ni terrestre, immortel ni mortel, pour que, tel un statuaire qui reçois la charge et l'honneur de sculpter ta propre personne, tu te donnes toi-même la forme que tu auras préférée. Tu pourras dégénérer en un de ces êtres inférieurs que sont les bêtes, tu pourras, selon les vœux de ton cœur, être régénéré en un de ces êtres supérieurs que l'on qualifie de divins. ${ }^{57}$

27 L'homme se voit attribuer la liberté fondamentale de déterminer sa nature, c'est à lui de se donner la «forme » qu'il aura choisie. Rabelais ouvre dans sa fiction le champ immense de la liberté humaine, il en fait d'ailleurs l'objet central du Tiers livre qui porte essentiellement sur cette terrible ouverture du libre arbitre ${ }^{58}$. Le foisonnement des formes, la tension permanente d'un imaginaire enclin à présenter la multitude des aspects du monde, replace sans cesse l'homme devant la difficulté et la nécessité de redéfinir inlassablement sa nature. Toujours en mouvement ${ }^{59}$, toujours menacée, la nature de l'homme n'est à aucun moment le fruit d'une conception essentialiste chez Rabelais. L'homme ne devient humain que s'il est pris dans une dynamique de 
confrontation, d'émulation. Cette rémanence des images animales, cet entêtement de la figure marginale à menacer l'ordre établi, sont les conditions sine qua non d'une esthétique de la libertéé, travaillant sans relâche, avec brutalité et éclat, la définition de l'homme ${ }^{61}$.

\section{NOTES}

1. "Les proverbes tirés du monde des animaux, surtout des animaux domestiques, sont nombreux et variés. C'est ici qu'éclate la forte originalité de la parémiologie rabelaisienne et qu'elle se révèle un cachet à part ». Lazare Sainéan, La Langue de Rabelais, Genève, Slatkine Reprints, 1976, p. 380.

2. Jacques Rancière, La chair des mots, Politiques de l'écriture, Paris, Galilée, 1998.

3. Lucien Febvre a noté cette « infirmité » de l'homme renaissant vis-à-vis de la vue. Selon lui, les écrivains du XVIème siècle «à de très rares exceptions près [...] ne savent pas faire un croquis, attraper une ressemblance, camper un personnage en chair et en os devant le lecteur ». Le Problème de l'incroyance au XVI" siècle. La religion de Rabelais, Paris, Albin Michel, «L'évolution de l'humanité ", (1942), 1961 et 1974, p. 79-81. Nous verrons que, pour singulières qu'elles fussent, les modalités esthétiques du voir constituent une problématique centrale chez Rabelais.

4. Michel Foucault, Les Mots et les choses Une archéologie des sciences humaines, «La Prose du monde », Paris, Tel, Gallimard, 1966, p. 50-51. Nous soulignons.

5. L'hidalgo passe pas moins de quatre jours pour trouver un nom à son roussin et huit jours pour se nommer lui-même. L'auteur met alors en abyme le travail de création et répète l'acte de la Genèse, inscrivant son œuvre dans la continuité - parodique - du geste primitif d'Adam : «[...] après plusieurs noms qu'il forma, effaça et ôta, ajouta, défit et refit en sa mémoire et imagination, enfin il vint à le nommer Rossinante, nom, à son avis, haut, sonore, et significatif de ce qu'il avait été quand il avait été roussin auparavant, et de ce qu'il était à présent, qui était devant et le premier de tous les roussins du monde ». Cervantès, L'ingénieux hidalgo Don Quichotte de la Manche, traduction de César Oudin, Paris, Folio classique, Gallimard, 1988, p. 71.

6. Voir infra: «Anti-nomination et figuration : de l'inversion du signe à sa matérialité » pour une analyse de cette liste.

7. La notion de stupeur - issue du latin stupor - traduit bien cette idée de saisissement, d'attente. Le lecteur reste interdit, paralysé par une image déconcertante, dont le sens lui est a priori refusé au profit d'une forme dominatrice et surinvestie du point de vue de la représentation. C'est bien la forme, l'image qui triomphe tandis que son assujettissement au rôle d'illustration s'estompe. Julie Jourdan prépare, sous la direction de Jean-Claude Schmitt, une thèse sur les rapports entre le texte et l'image dans le Ci nous dit. Elle y développera notamment l'idée d'une « rhétorique de la stupeur ».

8. Dans le dialogue de Platon, Cratyle, le philosophe éponyme soutient que le mot correspond à l'être. Selon lui, le mot est intrinsèquement lié à la chose. D'après cette conception du langage, il est impossible de tenir un discours faux. Nous ne disons pas que Rabelais fût un disciple de Cratyle, bien au contraire (il disait bien que les mots signifiaient «à plaisir», selon une convention et non par nature, voir livre, chapitre XIX, p. 409 - éd. Pléiade - : « Les langaiges sont par institutions arbitraires et convenences des peuples : les voix (comme disent les Dialecticiens) ne signifient naturellement, mais à plaisir »), mais nous avançons l'hypothèse d'un imaginaire 
proche de cette conception philosophique, un imaginaire qui compte sur la force d'évocation des mots, dans leur simple matérialité sonore.

9. Jean-Claude Schmitt, «Imago : de l'image à l'imaginaire », Cahiers du Léopard d'or 5, 1996, p. 31. 10. Ce statut primordial de la vue chez Rabelais est particulièrement notable, dans la mesure où les chercheurs, à la suite de Lucien Febvre, avaient eu tendance à minorer ce sens chez les écrivains de la Renaissance: "Comme l'ouïe fine et le flair aiguisé, les hommes de ce temps avaient, sans nul doute, la vue perçante. Mais précisément, ils ne l'avaient pas encore mise à part des autres sens. Ils n'en avaient pas lié spécialement les données par un lien nécessaire, à leur besoin de connaître [...]». Lucien Febvre, Le Problème de l'incroyance au XVIème siècle, op. cit., p. 79-81. Michel Simonin fait état de cette lecture dénigrant la vue dans les œuvres de la Renaissance, notamment chez un historien tel que Robert Mandrou qui parle du "rôle secondaire de la vue ", afin de démontrer qu'il n'en est rien. M. Simonin s'appuie cependant sur des textes plus tardifs, et délaisse le cas de Rabelais. "Le statut de la description à la Renaissance ", in L'Encre et la lumière Quarante-sept articles (1976-2000), Genève, Droz, 2004, p. 163-177.

11. Gargantua, Prologue de l'auteur, édition de Mireille Huchon, Paris, Pléiade, Gallimard, 1994, p. 6. Nous soulignons. Nos références au texte de Rabelais sont tirées de l'édition Pléiade établie par Mireille Huchon, hormis dans le dernier volet de cet article : « Une figure marginale : Panurge eschappé es chiens », pour lequel nous avons utilisé l'édition princeps établie par V. L. Saulnier.

12. Tiers livre, Prologue de l'Auteur, p. 345. Nous soulignons.

13. Index électronique des œuvres romanesques (les cinq livres de Pantagruel) de François Rabelais, sous la direction de Marie-Luce Demonet. Index et édition publiés avec le concours de l'Université Blaise Pascal et du Centre National du Livre, Tours, La Licorne, 1999.

14. Michel Charles, «Une rhapsodie herméneutique », in Rhétorique de la lecture, Paris, Edition du Seuil, 1977, p. 33-58.

15. «La « pusse en l'oreille » enchâssée et montée en bijou au Tiers livre (chapitre VII), symbolise certes l'inquiétude de Panurge en proie à ses appétits sensuels, mais elle représente aussi, au niveau métalinguistique, le souci de traduire visiblement cette sensualité poignante que l'usure ou la banalité du proverbe n'auraient su transmettre». François Rigolot, "Sémiotique de la sentence et du proverbe chez Rabelais ", Etudes rabelaisiennes XIV, 1977, p. 277-286. Nous soulignons.

16. «[...] l'analogisme est un rêve herméneutique de complétude qui procède d'un constat d'insatisfaction prenant acte de la segmentation générale des composantes du monde sur une échelle de petits écarts, il nourrit l'espoir de tisser ces éléments faiblement hétérogènes en une trame d'affinités et d'attractions signifiantes ayant toutes les apparences de la continuité ». Philippe Descola, «Les vertiges de l'analogie », in Par-delà nature et culture, Paris, nrf, Gallimard, 2005, p. 280. Nous soulignons.

17. «Pour louer Socrate, messieurs, je procéderai par comparaison ; lui croira peut-être que je veux le tourner en ridicule; non, c'est un portrait réel et non une caricature que je veux tracer ainsi. Je dis donc qu'il ressemble tout à fait à ces silènes qu'on voit exposés dans les ateliers des statuaires, et que l'artiste a représentés avec des syringes et des flûtes à la main; si on les ouvre en deux, on voit qu'ils renferment à l'intérieur des statues de dieux. Je soutiens aussi qu'il ressemble au satyre Marsyas. Que tu ressembles de figure à ces demi-dieux, Socrate, c'est ce que toi-même tu ne saurais contester ; mais que tu leur ressembles aussi pour le reste, c'est ce que je vais prouver. Tu es un moqueur, n'est-ce pas? Si tu n'en conviens pas, je produirai des témoins. Mais je ne suis pas joueur de flûte, diras-tu. Si, tu l'es, et beaucoup plus merveilleux que Marsyas ». Platon, Le Banquet, 215a et b, traduction d'Emile Chambry, Paris, Classique Garnier, 1922.

18. Le mythe des Harpyes est fondé par Hésiode dans sa Théogonie à partir du vers 265 : «Quant à Thaumas le Merveilleux, ce fut une fille d'Océanos au cours profond / qu'il emmena dans sa 
demeure : Electre la Brillante; et celle-ci enfanta la rapide Iris, l'Arc-en-ciel, / et les Harpyes Ravisseuses aux beaux cheveux, Aellô la Rafale et Ocypétè Vol-Vif, / qui peuvent talonner les souffles des vents et des oiseaux [...] ». Traduction d'Annie Bonnafé, Paris, Rivages, 1993.

19. Nous nous appuyons ici sur les définitions de Pierre Grimal : «On représente [les Harpyes] comme des femmes pourvues d'ailes ou encore comme des oiseaux à tête féminine. Elles ont des serres aiguës. [...] Les Harpyes sont des ravisseuses d'enfants ou d'âmes. On plaçait parfois leur image sur les tombeaux, emportant l'âme du mort dans leurs serres ». / "Les satyres, appelés aussi "Silènes ", sont des démons de la nature, qui ont été intégrés dans le cortège de Dionysos. On les représentait de différentes façons : tantôt le bas du corps était celui d'un cheval et, à partir de la taille, le haut était celui d'un homme. Tantôt, leur animalité était celle d'un bouc. Dans l'un et l'autre cas, ils sont doués d'une longue et large queue, très fournie, semblable à celle d'un cheval, et d'un membre viril perpétuellement dressé de proportions surhumaines ». Dictionnaire de la mythologie grecque et romaine, Paris, PUF, 1951.

20. Michael Camille, Images dans les marges Aux limites de l'art médiéval, traduction de Béatrice et Jean-Claude Bonne, Paris, Le temps des images, Gallimard, 1997 (édition originale 1992), p. 11. Nous soulignons. Ici, M. Camille répond, semble-t-il, à Emile Mâle qui ne voyait dans l'art religieux du XIII ${ }^{e}$ qu'un miroir de la nature se réduisant à deux fonctions : l'une symbolique, l'autre laudative. E. Mâle ne concevait pas la possibilité d'un art subversif, ayant pour but de troubler la frontière de l'humain: "Aucune idée dans cet art charmant, mais un tendre et profond amour pour la nature. Les sculpteurs du Moyen Âge, livrés à eux-mêmes, ne s'embarrassaient plus de symboles : ils redevenaient peuple, ils regardaient le monde avec des yeux émerveillés d'enfant " L'Art religieux du XIIle siècle en France (1899), Paris, Le Livre de Poche, Armand Colin, 1993, p. 109-110.

21. Erasme, Traité de civilité puérile (1530), traduction d'Alcide Bonneau (1877), Paris, Mille et une nuit, Fayard, 2001 : « Nous en dirons autant de ceux qui rient en ouvrant horriblement la bouche, en se plissant les joues et en découvrant toute la mâchoire : c'est le rire d'un chien ou le rire sardonique », p. 15 ; «S'ingurgiter, d'un coup, de gros morceaux, c'est le fait des cigognes ou des goinfres ", p. 34. Le dessein de cet ouvrage est clairement éducatif et pédagogique, tout différent de celui des chroniques rabelaisiennes qui n'auraient pour objet affiché que de faire rire. Mais Rabelais instaure un dialogue facétieux entre ce petit livre et son Gargantua. Il fait volontairement suivre par son géant une pente grossière et bestiale, pour mieux fonder ensuite une éducation pleine d'appétit et de désir d'apprendre. Toujours rappelé à sa condition animale, l'homme doit sans cesse refonder son humanité.

22. Quartlivre, XXIX, p. 607.

23. T. Cave, Cornucopia Figures de l'abondance au XVI siècle: Erasme, Rabelais, Ronsard, Montaigne, Paris, Macula, 1997, p. 230.

24. Quart livre, XXX, p. 610.

25. Quart livre, XXXII, p. 613 (nous sautons les lignes utilisant des images autres qu'animales, mais respectons l'ordre de l'énumération).

26. Rabelais joue ici sur le double sens de «contenence ». Au chapitre XXII du Pantagruel de 1542, le mot désigne aussi bien l'attitude de la dame que les déjections des chiens : «Tout le monde se arestoit à ce spectacle considerant les contenences de ces chiens qui luy montoyent jusques au col, et luy gasterent tous ces beaulx acoustremens ", p. 297.

27. Expression de Floyd Gray dans Rabelais et le comique du discontinu, Paris, Honoré Champion Editeur, 1994.

28. Quart livre, XXXII, p. 614.

29. Quart livre, XXXII, p. 615.

30. En ceci, ces images sont très proches des images marginales telles que Gil Bartholeyns, Pierre-Olivier Dittmar et Vincent Jolivet les ont analysées : «Entre autres spécificités, le texte littéraire ou l'œuvre d'art refuserait la transparence du signifiant, provoquerait son étrangeté. Il 
interposerait un signifiant opacifié comme un écran devant le mondain, et suggérerait par là qu'il y a du monde [les auteurs reprennent ici des notions de Georges Molinié : le mondain se rapporte à tout ce qui est appréhendable, tandis que le monde désigne la brutalité du réel]. Les sensations nous paraissent traduites dans le texte littéraire ou dans l'image par l'attention portée à la matérialité du signifiant: effet de surface de l'ornement, sonorité du verbe... Cette matérialité qui résiste, sur laquelle nous butons, nous projette vers un au-delà de la signification où le sensible féconde le signifié ". "La transgression dépassée », in Image et transgression au Moyen Âge, Paris, PUF, 2008, p. 164-165. Nous soulignons.

31. Gil Batholeyns, Pierre-Olivier Dittmar, Vincent Jolivet, Image et transgression au Moyen Âge, op. cit., p. 165. Nous soulignons.

32. Quart livre, IIII, p. 546-547. Nous soulignons.

33. Quart livre, «A mon seigneur Odet, cardinal de Chastillon », p. 519.

34. Selon Roland Barthes, la littérature occupe une place privilégiée parmi les disciplines, dans la mesure où elle «fait tourner les savoirs » : «Elle n'en fixe, elle n'en fétichise aucun; elle leur donne une place indirecte et cet indirect est précieux. D'une part, elle permet de désigner des savoirs possibles - insoupçonnés, inaccomplis; la littérature travaille dans les interstices de la science : elle est toujours en retard ou en avance sur elle [...]. D'autre part, le savoir qu'elle mobilise n'est jamais entier ni dernier : la littérature ne dit pas qu'elle sait quelque chose, mais qu'elle sait de quelque chose ; ou mieux : qu'elle en sait quelque chose - qu'elle en sait long sur les hommes ». Leçon (leçon inaugurale au Collège de France prononcée le 7 janvier 1977), Paris, Seuil, 1989, p. 18-19. Nous soulignons.

35. Pantagruel, VIII. «Somme que je voye un abysme de science : car doresnavant que tu deviens homme et te fais grand, il te faudra yssir de ceste tranquillité et repos d'estude [...]», p. 245 . Force est de relever l'ambivalence de l'expression «abysme de science» dans cette lettre de Gargantua à son fils, traditionnellement présentée comme un morceau du plus pur optimisme humaniste. Je remercie ici Michel Magnien qui avait développé, pendant l'année universitaire 2004-2005, lors de son séminaire portant sur "L'ironie à la Renaissance », l'idée du caractère suspicieux de ce terme "abysme ». Il l'avait alors interprété comme un "signe ironique " (en s'appuyant sur la notion d'« ironie littéraire ", établie par Philippe Hamon), allant de ce fait à l'encontre des lectures habituelles du chapitre VIII de Pantagruel. M. Magnien s'était alors appuyé sur l'article de G. J. Brault : « "Ung abysme de science »: on the interpretation of Gargantua's letter to Pantagruel », Travaux d'humanisme et de Renaissance, tome XXVIII, Genève, Droz, 1966, p. 615-632.

36. Quart livre, LXIV, p. 691-692.

37. Christ du portail central de la cathédrale d'Amiens. Ce Christ écrasant des symboles maléfiques s'inscrit dans un système de représentation pleinement normatif. Il s'agit d'une image centrale, en majesté, point de mire du regard, au seuil de la cathédrale. Les animaux symboliques sont directement appréhendés en tant que tels. Cette immédiateté, cette transparence du signe est totalement renversée dans la liste du chapitre LXIV. Il s'agit d'une démarche esthétique absolument (volontairement ?) antinomique, qui brouille les usages traditionnels du symbole.

38. Michel Pastoureau a insisté sur cette lisibilité du symbole qu'il reste à interpréter : «[...] la pensée analogique médiévale s'efforce d'établir un lien entre quelque chose d'apparent et quelque chose de caché ; et, principalement, entre ce qui est présent dans le monde d'ici-bas et ce qui a sa place parmi les vérités éternelles de l'au-delà. Un mot, une forme, une couleur, une matière, un nombre, un geste, un animal, un végétal et même une personne peuvent ainsi être revêtus d'une fonction symbolique et par là même évoquer, représenter ou signifier autre chose que ce qu'ils prétendent être ou montrer ". Une histoire symbolique du Moyen Âge occidental, Paris, Seuil, 2004, p. 17. Nous soulignons. Justement, dans cette liste, les termes sont (pour la plupart) cachés, dans la mesure où ils constituent des transcriptions directes et souvent altérées du grec 
et de l'arabe. Pour les origines précises de ces mots, voir l'étude de Paul Delaunay, «Les Animaux venimeux dans Rabelais ", in Mélanges offerts à M. Abel Lefranc, Paris, Droz, 1936.

39. Pantagruel, IX, p. 246.

40. Lors de son intervention du 18 février 2009 au séminaire d'«Anthropozoologie : histoire naturelle et culturelle des animaux vrais ", intitulée: "Aux origines du bestiaire héraldique (Xème-XIIIème siècle) ", Michel Pastoureau a insisté sur les connotations foncièrement obscènes qui sont attachées au chien jusqu'à la fin du Moyen Âge. C'est seulement au cours de la Renaissance que les «cochonneries sexuelles" seront attribuées au cochon. On observera que cette symbolique luxurieuse du chien perdure fortement chez Rabelais et chez ses contemporains - notamment chez le juriste André Tiraqueau.

41. Pantagruel, IX, p. 246.

42. Sur la " grâce animale " voir les développements de Heinrich von Kleist dans son court récit Sur le théâtre de marionnettes: "Nous voyons que, dans le monde organique, plus la réflexion paraît faible et obscure, plus la grâce est souveraine et rayonnante ", Paris, édition Mille et une nuits, 2003, p. 20.

43. Pantagruel, chapitre IX, édition de 1532 établie par V. L. Saulnier, Genève, Librairie Droz, 1965. p. 49. Nous utiliserons à présent cette édition, car celle de la Pléiade n'offre pas la version première du texte. On lit également dans cette série de questions une parodie d'un vers formulaire de l'épopée homérique, celui de l'identité (Odyssée, I, 79). On trouve déjà ce détournement burlesque du vers homérique dans la cinquième section de l'Apocoloquintose [transformation en citrouille] $d u$ divin Claude, apothéose comique et satirique de l'empereur romain. Sénèque l'utilisait pour jeter le trouble sur l'identité de l'empereur lors de son arrivée dans l'olympe : "[Hercule] vit cette face d'une espèce nouvelle, cette démarche insolite, il ouït cette voix qui n'appartenait à aucun animal terrestre, qui n'était, comme chez les monstres marins, qu'un rauque et sourd grognement, et il pensa que le treizième de ses travaux lui tombait sur les bras. En y regardant mieux, il crut démêler quelque chose d'un homme. Il s'approcha donc et, chose facile à un roitelet grec, il débita ce vers d'Homère : «Quel es-tu? D'où viens-tu? De quel pays es-tu?»". Euvres complètes de Sénèque le Philosophe, traduction de J. Baillard, Paris, Hachette, 1914, premier volume, p. 269. De la même façon, Rabelais reprend ce vers pour mieux souligner l'identité incertaine de Panurge, ses rapports équivoques avec les différentes catégories de la Création.

44. Pantagruel, chapitre XIV, p. 124.

45. Ibid.

46. "Je ne t'ai pas vu, vaurien, tendre des pièges au bouc de Damon, malgré les aboiements de Lycisca?». Bucoliques, III, 16-18, texte établi par Eugène de Saint-Denis, Paris, «Les Belles lettres », 1956.

47. Pantagruel, chapitre XIV, p. 122.

48. Rabelais, Euvres complètes, Pantagruel, p. 195.

49. «Mais il ajoute que cette impudence des femmes (dont nous parlons plus bas lorsque nous traitons du caractère envieux des femmes) a fait que celles-ci ont été comparées par beaucoup aux chiens, parce que rien n'est plus impudent que cet animal. Le divin Jérôme rapporte le fait, dans le livre d'Esaïe, au chapitre 66. C'est de belle façon (dit-il) que le chien et la prostituée s'unissent, car l'un et l'autre animal sont enclins au désir libidineux », (nous traduisons) André Tiraqueau, « canum nomine vocantur impudentes », De legibus connubialibus (Anvers, 1530), liber 9, numero 183, Lyon, apud Guliel Rovilluim, 1554.

50. Jean-Claude Schmitt a mis en avant le rôle structurant du paradigme de la dissemblance dans les systèmes figuratifs de la Chrétienté : «Le ad de la formule biblique [«Faciamus hominem ad imaginem et similitudinem nostram » : "Faisons l'homme à notre image et notre semblance ", Genèse, I, 26] indique que, pour l'homme, cette histoire est projet, le projet d'une restitution pleine de la « ressemblance » perdue qui ne demeure qu'à l'état de trace (vestigium) dans l'état 
de « dissemblance » et d'éloignement de Dieu où l'homme, par la Faute, s'est lui-même plongé. Dans ce drame qui est, dans la conception chrétienne, le fondement de toute histoire, l'homme incarne le changement alors que Dieu est l'être immuable ». Le Corps des images Essais sur la culture visuelle au Moyen Âge, Paris, « Le Temps des images », nrf, Gallimard, 2002, p. 23.

51. André Tiraqueau, De Legibus connubialibus, «canis in propatulo coit », liber 15, numero 159, op. cit.

52. «L'univers caustique des marges emprunte volontiers au registre de l'obscène. En regard des textes sacrés, cette obscénité peut être perçue comme blasphématoire. Or, la juxtaposition de registres à ce point contraires n'est manifestement pas transgressive au Moyen Âge (du moins pas dans sa conception), car cette juxtaposition des images marginales et du centre est ordonnée, organisée, hiérarchique [...]». "Montages normatifs", in Image et transgression au Moyen Âge, op. cit., p. 81. Nous soulignons. Selon les auteurs de ce livre : «Seule l'abolition de hiérarchie entre marges et centre, c'est-à-dire l'intimité d'éléments disparates, rendrait incertaines les frontières entre les catégories, brouillerait leur lisibilité, ébranlerait l'ordre » (p. 85). Or, c'est bien ce qui se passe dans la fiction rabelaisienne où la figure marginale de Panurge renverse les catégories, fait verser le plus haut, le plus noble, le plus solennel, dans le plus bas, le plus infâme. Il n'apporte pas un contrepoint burlesque à un univers officiel et ordonné. Il ne s'inscrit pas dans un rite carnavalesque (des fêtes marginales pouvaient être associées à la procession de la Fête Dieu), puisqu'il habite le sein de la norme, et le sape littéralement.

53. Voir à ce propos les travaux de Jean Scheidegger, Le Roman de Renart ou le texte de la dérision, Genève, Droz, 1989. L'auteur parle à propos de l'attitude de Renart de "négativité radicale ", cependant, il relève la contiguïté entre la renardie et l'écriture, et ajoute : « Mais de sa négation et de la chose niée [...] reste quelque chose. La négativité renardienne fait œuvre, elle est à l'œuvre. La dérision maîtrisée, opérée avec maîtrise par le roman, constitue et aménage un espace littéraire " (p. 364-365). Cet espace littéraire aménagé par Renart acquiert une certaine stabilité malgré l'évolution des branches partant d'un XII ${ }^{\mathrm{e}}$ siècle anomal et allant vers un XIII siècle nettement plus allégorique et moralisant. De fait, l'univers renardien est constitué d'une certaine homogénéité, la figure de Renart reste stable et reconnaissable, tandis que l'univers qui se déploie autour de lui n'a de raison d'être que son renversement malicieux ou maléfique. L'hétérogénéité structurelle des chroniques rabelaisiennes ouvre un espace littéraire bien plus difficile à appréhender, où la norme côtoie la marge, où la farce malmène l'érudition la plus sérieuse, où le mal s'abolit en éclat de rire, où les symboles s'égarent en des structures inouïes.

54. Paul Radin a établi les caractéristiques de la figure du «trickster » qui se définit par une ruse sans limites, qui passe tous les codes, toutes les conventions, toutes les lois. Voir Regan Paul, The trickster: a study in american indian mythology, Londres, Routledge, 1956. L'article de J. P. Bordier «Pathelin, Renart, Trubert, décepteurs " (in Le Moyen Âge 98, 1992, p. 71-84) inscrit de grandes figures comiques du Moyen Âge français dans les particularités de ce type.

55. Le centon est une étoffe ou un vêtement fait de plusieurs morceaux de couleurs différentes. Par analogie, on parle en littérature de centon, lorsqu'une pièce de vers ou de prose est composée de passages empruntés à un ou plusieurs auteurs (Source: TLFi, Trésor de la Langue Française informatisé). Sur l'histoire de l'usage littéraire du centon, voir l'article d'Hélène Cazes : «Centon et collage: l'écriture cachée ", in Montages / Collages, Actes du second colloque du CICADA, Publications de l'Université de Pau, 1993, p. 69-84.

56. Corneille Agrippa, De incertitudine et vanitate scientiarum et artium, atque excellentia verbi dei declamatio, Parisiis, J. Petrum, 1531. Cet ouvrage marque un tournant dans la pensée d'Agrippa qui passe de l'occultisme le plus profond à un fidéisme qui le conduit à remettre en cause les capacités de l'homme à connaitre le monde. Ce scepticisme a certainement influencé la pensée de Rabelais. 
57. Pic de la Mirandole, Discours de la dignité de l'homme (1486), traduction du latin par Roland Galibois, texte reproduit par Jean-Christophe Saladin, dans la Bibliothèque humaniste idéale De Pétrarque à Montaigne, Paris, Les Belles Lettres, 2008, p. 126-127.

58. Le dilemme de Panurge (Dois-je me marier ou pas ?) incarne dans la fiction rabelaisienne cette difficulté consubstantielle à la liberté : celle de devoir accepter la prise de risque. La peur de Panurge résume sans ambages cet immense défi que constitue l'avancée vers la connaissance de soi ; Pantagruel le lui signifie clairement: «N'estez vous asceuré de vostre vouloir? Le poinct principal y gist : tout le reste est fortuit, et dependent des fatales dispositions du Ciel ». Tiers livre, chapitre X, op. cit., p. 379-380.

59. Sur cette importance du mouvement dans l'esthétique de la Renaissance, voir l'ouvrage de Michel Jeanneret Perpetuum mobile Métamorphoses des corps et des œuvres de Vinci à Montaigne, Paris, Macula, 1997, et la fameuse étude de Jean Starobinski Montaigne en mouvement, Paris, Gallimard, 1982.

60. Il s'agit bien d'une esthétique de la liberté et non d'une éthique, dans la mesure où l'éthique impliquerait une dimension normative. Il n'en est rien chez Rabelais qui échappe à toute idéologie. Il travaille avec ses armes de créateur, place des images, aménage un espace de confrontation, dispose, propose, inquiète, à la manière du " poisson torpille » socratique (Ménon, 80a), mais n'impose jamais un système de pensée établi.

61. Cet article achevé, il me faut à présent témoigner ma plus vive reconnaissance à Elisa Brilli, Pierre-Olivier Dittmar et Jean-Claude Schmitt qui ont la patience et la générosité de m'initier aux problématiques de l'iconographie médiévale.

\section{RÉSUMÉS}

Cet article offre un travail analytique sur l'une des figures structurantes de la poétique rabelaisienne : l'analogie. Il s'agit de mettre en évidence les particularités de cette figure dans l'économie narrative et descriptive du poète renaissant. Afin d'offrir une vision appréciable de ce procédé poétique, cette étude ce concentre sur un champ restreint de l'imaginaire : l'animalité. L'analogie entre la bête et l'homme pose des problèmes spécifiques et fondamentaux qui interrogent le concept antique d'humanitas. Il s'agit de comparer l'esthétique de Rabelais avec celle à l'oeuvre dans l'art médiéval, et de comprendre le jeu de l'auteur avec la tradition symboliste et analogiste. Cette dynamique comparatiste confrontant les problématiques de l'image textuelle à celles de l'image matérielle débouche sur une réflexion anthropologique et philosophique : comment Rabelais «champion de l'humanisme » conçoit-il l'homme, comment appréhende-t-il ses pouvoirs ? L'incertitude, la fragilité, le mouvement, le devenir apparaissent, en dernière instance, comme les garanties d'une conception vraie de l'homme - toujours inquiétée, toujours refondée.

\section{INDEX}

Mots-clés : analogisme, animal, Descola (Philippe), enluminure, humanisme, Rabelais (Français) 


\section{AUTEUR}

\section{LOUISE MILLION}

Louise Millon est professeur agrégé de Lettres modernes, doctorante de l'Université de la Sorbonne nouvelle et prochainement de l'Ecole des hautes études en sciences sociales. Elle mène ses recherches sur l'imaginaire animal de François Rabelais, et ouvre cette réflexion littéraire sur l'iconographie médiévale du XIII ${ }^{\mathrm{e}}$ au XVI ${ }^{\mathrm{e}}$ siècle, afin de constituer une sorte d'archéologie des motifs animaliers partant du champ textuel de Rabelais pour s'étendre à différents domaines de l'art médiéval (marginalia et statuaire en particulier). Co-dirigée par Marie-Madeleine Fragonard et Jean-Claude Schmitt, elle suit une méthode comparatiste interrogeant les rapports entre les images matérielles et les images verbales. 